1 Title: The maternal foam plug constitutes a reservoir for the desert locust's bacterial

\title{
2 symbionts
}

3 Running title: Foam-mediated bacterial transmission in locusts

4 Author names and affiliation: Omer Lavy ${ }^{1}$, Uri Gophna* ${ }^{2}$, Amir Ayali* ${ }^{1}$, Shalev Gihaz ${ }^{3}$,

5 Ayelet Fishman ${ }^{3}$, \& Eran Gefen* ${ }^{4}$

6 (1) School of Zoology, Tel Aviv University, Tel Aviv, Israel

7 (2) School of Molecular Cell Biology and Biotechnology, Tel Aviv University, Tel

$8 \quad$ Aviv, Israel

(3) Department of Biotechnology and Food Engineering, Technion-Israel Institute of

10 Technology, Haifa, 3200003, Israel

(4) Department of Biology, University of Haifa - Oranim, Kiryat Tivon, Israel

12 E-mail addresses according to authors order: Omer.Lavy@gmail.com,

13 urigo@tauex.tau.ac.il, ayali@tauex.tau.ac.il, shalevgihaz@gmail.com,

14 afishman@technion.ac.il, gefene@ research.haifa.ac.il

$15 *$ Corresponding authors:

16 Uri Gophna: urigo@tauex.tau.ac.il, Phone: +972-36409988, Fax: +972-36409407, Tel

17 Aviv University, P.O. Box 39040, Tel Aviv 6997801, Israel.

18 Amir Ayali: ayali@ tauex.tau.ac.il, Phone: +972-3640576, Fax: +972-36409403, Tel Aviv

19 University, P.O. Box 39040, Tel Aviv 6997801, Israel. 
20 Eran Gefen: gefene@ research.haifa.ac.il , Phone: +972-4-9838837, Fax:+ 972-49539608,

21 Department of Biology, Faculty of Natural Sciences, University of Haifa - Oranim, Tivon

223600600 , Israel.

\section{Abstract:}

25 A hallmark of the desert locust's ancient and deserved reputation as a devastating 26 agricultural pest is that of the long-distance, multi-generational migration of locust

27 swarms to new habitats. The bacterial symbionts that reside within the locust gut 28 comprise a key aspect of its biology, augmenting its immunity and having also been

29 reported to be involved in the swarming phenomenon through the emission of attractant

30 volatiles. However, it is still unclear whether and how these beneficial symbionts are 31 transmitted vertically from parent to offspring. Using comparative 16S rRNA amplicon 32 sequencing and direct experiments with engineered bacteria, we provide here evidence of

33 the vertical transmission of locust gut bacteria. The females perform this activity by way

34 of inoculation of the egg-pod's foam plug, through which the larvae pass upon hatching.

35 Furthermore, analysis of the biochemical structure of the foam revealed chitin to be its

36 major component, along with immunity-related proteins such as lysozyme, which could

37 be responsible for the inhibition of some bacteria in the foam while allowing other, more

38 beneficial, strains to proliferate. Our findings reveal a potential vector for the

39 transgenerational transmission of symbionts in locusts, which contributes to the locust

40 swarm's ability to invade and survive in new territories. 


\section{Introduction:}

43 Locusts (order: Orthoptera) have affected the lives of people at least since biblical times

44 (Old Testament: Exodus 10: 4-19), and still constitute a serious agricultural threat today

45 (FAO 2020; Zhang et al., 2019). During locust outbreaks or plagues, these highly

46 polyphagous insects (Chapman \& Joern 1990) perform long-distance migrations,

47 devastating agriculture in large parts of the developing world (FAO 2020; Symons \&

48 Cressman 2001; van Huis et al., 2007; Cease et al., 2015). The desert locust (Schistocerca

49 gregaria) is a well-known locust species, mostly originating in the African Sahel

50 (Symons \& Cressman 2001; Cease et al., 2015; Lorenz 2009). Under the appropriate

51 conditions, S. gregaria swarms develop and can potentially reach the Arabian Peninsula,

52 the Middle East, southern Europe, and even south-west Asia (FAO 2020).

53 These large-scale migrations comprise several consecutive generations (Symons \&

54 Cressman 2001; Skaf et al. 1990). Each generation develops through five nymphal instars

55 into reproductive adults ((Symons \& Cressman 2001). Post-copulation, females oviposit

56 their egg pods within the soil, enveloping the eggs in a fine sheath of foamy secretion of

57 previously unknown composition. The same secretion is deposited as a thick foam plug

58 above the egg pods (Fig. 1). Upon hatching the young hatchlings crawl through the foam

59 plug to reach the soil surface and start a new locust generation (Symons \& Cressman

60 2001; Uvarov 1997; Hägele et al., 2000).

61 An important aspect of the desert locust biology is that of its symbiosis with the bacteria

62 inhabiting its hindgut. These bacteria have been shown to augment the locust's immunity

63 through colonization resistance (Dillon \& Charnley 1995, 2002; Dillon et al., 2000,

64 2005), and were also suggested to contribute to maintaining the swarm's integrity 
65 through the emission of attractant volatiles (Dillon \& Charnley 2002; Dillon et al., 2000,

66 2002). Although S. gregaria does not engage in an obligate interaction with any specific

67 bacterial species, it is consistently associated with bacteria of the families

68 Enterobacteriaceae and Enterococcaceae (Dillon \& Charnley 2002, Shi et al., 2014;

69 Lavy et al., 2019, 2020). It has been traditionally believed that the locust's acquired

70 bacteria are strictly environmentally-determined (Dillon \& Charnley 2002; Salem et al.,

71 2015). However, migrating $S$. gregaria in their swarming phase encounter a variety of

72 different environnements, plants, and oviposition sites, which vary in terms of the

73 bacteria to which the locusts are exposed (Cease et al., 2015; Uvarov 1977; Popov 1958;

74 Pener \& Simpson 2009; Soussi et al., 2015). Hence, it is unlikely that the habitat of the

75 newly-hatched individuals can be the sole source of the bacterial agents that eventually

76 play the pivotal physiological roles noted above. Rather, we hypothesized that the locusts

77 possess a core microbiome that is vertically transmitted across generations; and that the

78 effect of such a mechanism could be amplified through a selectivity trait of the locusts or

79 through the transmission mode of the symbionts.

80 There are numerous examples of insects that inoculate their offspring with beneficial

81 bacteria, through different mechanisms. The burying beetle (Nicrophorus vespilloides),

82 for example, manipulates the bacterial composition of the carcasses upon which its larvae

83 are reared (Shukla et al., 2017), and inoculates its young with advantageous gut bacteria

84 (Wang \& Rozen 2017, 2018). Other examples include brood-cell smearing with

85 protective vertically transmitted Streptomyces by digger wasps (Kaltenpoth et al.,

86 2005,2019; Koriss et al., 2010; Engl et al., 2018); and symbiont-enclosing capsules

87 deposited by Plataspidae (suborder: Heteroptera) females to enable symbiont acquisition 
88 by their hatchlings (Fukatsu \& Hosokawa 2002). These and other inoculation

89 mechanisms have been thoroughly reviewed in the past (Salem et al., 2015; Onchuru et

90 al., 2018).

91 We recently reported the same operational taxonomic unit (OTU), assigned to

92 Enterobacter (Enterobacteriaceae), as being present in gregarious and solitary locusts

93 (both laboratory-reared) across several generations (as well as in field-collected $S$.

94 gregaria; Lavy et al., (2019)). This finding suggests transgenerational symbiont

95 inoculation in the desert locust. Here, we examined whether locusts vertically transmit

96 beneficial bacterial agents, and uncovered a mechanism by which bacteria may be

97 inoculated across generations, contributing to the successful migration of locusts to new

98 territories.

99 Results

100 Since it had been shown previously in the desert locust that bacterial agents are not 101 transmitted vertically through the germline (Charnley et al., 1985), we employed 16S 102 rRNA gene amplicon sequencing to compare the bacterial composition of gregarious $S$. 103 gregaria females to that of their offspring and to the immediate environment of their egg 104 pod (i.e. foam and sand) (data are openly available in the SRA archive: PRJNA598984).

105 In an attempt to explore uncover the possible routes of symbiont transmission, our first 106 analysis compared the bacterial composition of hatchling and pre-hatched siblings 107 derived from the same egg pod: hatchlings (newly-hatched locusts that had climbed to the 108 soil surface; n=16 egg pods), and pre-hatchlings (viable, healthy-looking embryos, 109 excavated minutes before their estimated hatching time; $\mathrm{n}=11$ egg pods). The individuals 
110 resulting from these two treatments have been shown to differ in both their bacterial

111 diversity (mean values of Shannon diversity index: 2.09 and 1.28 respectively; Fig. 2)

112 and in their overall bacterial composition (genus level, Bray-Curtis-based analysis of

113 similarities-'ANOSIM': $\mathrm{R}=0.15, p=0.024)$. Assuming that no bacterial transmission

114 occurs through the germ line (Charnley et al. 1985, Dillon and Charnley 2002) these

115 differences in bacterial composition found between the two treatments suggest that the

116 hatchlings acquire bacteria from their immediate environment post-hatching, with either

117 the foam or the surrounding sand as potential sources of inoculation. Moreover, the foam

118 samples ( $\mathrm{n}=15$ egg pods) showed a significantly higher bacterial diversity in comparison

119 to the sand samples ( $\mathrm{n}=16 \mathrm{egg}$ pods) (mean values of Shannon diversity index: 1.07 and

1201.74 respectively, Fig. 2), indicating the foam plug (through which the offspring must

121 crawl post-hatching), as a potential bacterial reservoir and source of inoculation.

122 Next, we identified 23 common amplicon sequence variants (ASVs) shared among the

123 hindgut of the females (i.e. the mothers), the foam plug and the hatchlings, including

124 sequences assigned to the genera Enterobacter, Klebsiella, and Corynebacterium, which

125 have been previously shown to be associated with S. gregaria (Dillon et al., 2002; Lavy

126 et al., 2019, 2020; Fig. 3b). Further analysis of the core genera (comprising the bacterial

127 composition of at least $80 \%$ of each sample type) revealed that while Corynebacterium

128 was prevalent in the foam and hatchling samples, it did not pass the threshold to be

129 considered as core bacterium in the hindgut (did not reach $>80 \%$ of the gut samples).

130 Other core bacteria prevalent in the female hindgut, such as Weissella, were absent from

131 both the foam and hatchlings (Fig 3). Nevertheless, a comparison of ASVs in full sets of

132 mothers and their specific foam plugs and offspring, revealed the presence of the same 
133 ASV assigned to the genus Corynebacterium (ASV 2), as being shared among these 134 samples in 10 out of 14 sets $(\sim 71 \%$, Table 1$)$.

135 Further paired-sample analysis of the same data revealed a significant correlation 136 between the relative abundance of Corynebacterium in specific foam plugs and the 137 hatchlings that had crawled through these plugs (Fig. 4). Such correlation of relative 138 abundance levels was not observed for the pre-hatching individuals and the foam plugs, 139 nor for the hatchlings and the sand surrounding them. These findings further suggested a 140 role for the foam plugs in the post-hatching bacterial acquisition, and that the genus 141 Corynebacterium is transmitted in such a manner.

142 To test the above hypothesis, we then compared the levels of Corynebacterium in foam143 deprived hatchlings ("without foam" treatment; $n=18$ egg pods), which were manipulated 144 to experience only the surrounding sand post-hatching, with that in their non-manipulated 145 siblings ("with foam" treatment; $\mathrm{n}=19$ egg pods). The latter showed significantly higher 146 levels of Corynebacterium post-hatching (Fig. 5). This validated the above-noted 147 hypothesis and also supported the hypothesized role of the foam in the vertical 148 transmission of these bacteria.

149 The next step in confirming our hypothesis would have been to demonstrate in-vivo the 150 inoculation of the foam with Corynebacterium. However, despite substantial efforts, we 151 were unable to isolate any locust-associated Corynebacterium from any of the animals. 152 Therefore, in order to directly test the possibility of the female gut as a source of the foam 153 bacteria, we inoculated locust females with a strain of Klebsiella pneumoniae isolated 154 from locust feces. We genetically engineered that strain (see Methods) to contain two 155 selectable markers that confer resistance to the antibiotics kanamycin and streptomycin. 
156 That resistant Klebsiella strain was found to be present in the inoculated female's feces 7

157 days post-inoculation. Consequently, we concluded that it was stably maintained in the

158 female gut and we then allowed these locusts to lay eggs in sterilized sand. On day 1

159 post-oviposition this Klebsiella strain was found in 11 out of 15 foam plugs secreted by

160 these females, but in only 3 samples of the sand surrounding the egg pods. The

161 engineered Klebsiella strain was still detectable in five foam plugs and two sand samples

162 after 10 days.

\section{Chemical analyses}

164 The absence of Weissella (which was very dominant in the female gut; Fig. 3a) in the 165 foam, combined with the constant presence of Corynebacterium and other locust 166 associated bacteria, such as Klebsiella (Fig. 3a) in the plugs, suggested some bacterial 167 selectivity traits of the foam. To determine whether the foam plug might possess 168 biochemical properties that select for particular microbes, we analyzed its composition.

169 We determined the foam plugs' total protein content by elemental analysis, and identified 170 the protein repertoire using SDS-PAGE and MS analysis. Elemental analysis of the foam

171 plug (Table. 2) indicated the presence of $\sim 0.63 \%$ nitrogen, which is translated to a 3$1723.5 \%$ protein fraction. Nevertheless, this small protein component contained at least eight 173 proteins that, out of the 42 proteins that could be identified by mass spectrometry, could 174 be attributed to the locust's immune system, such as a thaumatin-like protein, 175 prophenoloxidase, and lysozyme (Tables 3), (Table. S3). The low protein content of the 176 foam suggests that it mostly comprises carbohydrates. However, the extremely stable and 177 hydrophobic nature of the foam prevented a carbohydrate profile analysis. Since the foam 178 is insect-derived, we hypothesized that these carbohydrate molecules are composed of 
179 chitin or a chitin-like polysaccharide. Treating the foam with chitinase (a chitin degrading

180 enzyme) caused it to lose its hydrophobicity and to change in shape and color (Fig. 6),

181 thus confirming our hypothesis that chitin was a major component of the plug, but

182 probably not the only component.

\section{Discussion}

184 The success of locust swarms in covering very large distances and conquering new 185 grounds during an upsurge (Symmons \& Cressman 2001; Skaf et al., 1990) may be, at 186 least partly, attributed to the locust's interaction with its beneficial gut microbes, which 187 are important for its immunity (Dillon \& Chrnley 1995, 2002; Dillon et al., 2005) and 188 may also be instrumental in maintaining locust aggregation behavior (Dillon \& Chrnley 189 2002; Dillon et al., 2000, 2002). Though much knowledge has been acquired concerning 190 the locusts' bacterial symbionts (Dillon \& Chrnley 2002; Shi et al., 2014; Lavy et al., 2019,2020), the mechanism that maintains these bacteria within the population and 192 possibly transmits them from one generation to the next, has remained unclear. The 193 commonly suggested hypothesis has been that locusts acquire their endosymbionts as 194 hatchlings from their environment (Dillon \& Charnley 2002). However, the importance 195 of these bacteria and the diversity of feeding and oviposition habitats that females 196 encounter while migrating (Popov 1958; Uvarov 1977; Pener \& Simpson 2009; Cease et 197 al., 2015), make it likely that they possess some sort of mechanism to ensure the 198 inoculation of their offspring with beneficial bacteria. Here, we have shown that $S$. 199 gregaria females deposit bacterial symbionts in the foam they secrete on top of their 200 eggs, which in turn seems to act as a selective reservoir of these bacteria, passively 201 inoculating the offspring post- hatching. 
202 Our data suggest that the locust hatchlings acquire these bacterial symbionts post203 hatching, while climbing to the surface through the foam plug. The bacterial ASVs, 204 shared among the female's gut, the foam, and the hatchlings, provide further evidence of 205 the maternal origin of these foam-inhabiting bacteria. These findings of post-hatching 206 inoculation are in agreement with the possibility of obtaining bacteria-free (axenic) 207 locusts through egg surface-sterilization (Charnley et al., 1985), indicating that germ line 208 transmission does not take place in this insect species.

209 However, our experiments indicate that the inoculation process is also somewhat 210 selective: Weissella, for example, which is highly prevalent in the mother's gut, was not 211 found either in the foam or in the offspring samples at the end of the incubation period 212 (11 days at $37^{\circ} \mathrm{C}$ in this case). It seems that during this period, factors in the foam 213 enabled specific bacterial strains to proliferate, while inhibiting others. The presented 214 data concerning the link between the Corynebacterium in the foam and that in the 215 hatchlings emerging through it, further supports this hypothesis of foam selectivity; 216 which also explains the difference in bacterial composition between the mother's hindgut 217 and the foam.

218 Although we failed to successfully cultivate locust-associated Corynebacterium strains, 219 we were able to demonstrate the transmission of a genetically-modified locust-isolated 220 Klebsiella strain from the female gut to the foam plug. Our results clearly indicate that 221 locust females inoculate the foam with gut bacteria upon oviposition, offering a possible 222 route for bacteria that originate in the female gut, to reach her offspring.

223 Both Enterobacter and Klebsiella, which we found to be shared among locust mothers 224 and offspring, were also consistently found as hindgut bacterial symbionts of the desert 
225 locust in previous studies, and species of both genera were found to produce guaiacol and

226 phenol,which are considered to be cohesion pheromones that help to maintain the

227 integrity of the swarm (Dillon et al. 2000; Dillon \& Charnley 2002). Moreover, we

228 previously demonstrated the consistency of the same Enterobacter lineage through

229 several generations of laboratory-reared locusts as well as field-collected S. gregaria

230 (Lavy et al., 2019). Those findings suggested the existence of a mechanism responsible

231 for maintaining these gut bacteria within the population, including a transgenerational

232 inoculation mechanism, such as the foam which was the focus of the present study.

233 Corynebacterium is known to inhabit insects of different orders (e.g. Zucchi et al., 2012;

234 Segata et al., 2016; Tobias 2016; Park et al., 2019), as well as the reproductive system of

235 locust females (Lavy et al., 2020). Itoh et al. (1996, 1997) identified several

236 Corynebacterium species that have the rare ability to utilize gaseous acetophenone as a

237 sufficient carbon source to ensure their proliferation. Acetophenone has also been

238 identified in the gaseous fraction emitted from foam plugs secreted by $S$. gregaria

239 females (Rai et al., 1997). It is thus plausible that the acetophenone in the foam enriches

240 Corynebacterium species that, in turn, can produce antibiotic substances (Tobias 2016;

241 Gumiel et al., 2015). The locusts thereby "feed" the bacteria that in turn contribute to

242 protecting the locusts' eggs.

243 However, this is not the only mechanism that locusts appear to utilize to ensure the

244 presence of specific bacteria in the foam. Mass-spectrometry analysis of the protein

245 fraction of the foam revealed several immune-related peptides, such as lysozyme, a

246 thaumatin-like protein, and prophenoloxidase. We speculate that the presence of these

247 peptides in the medium surrounding the eggs provides an extra layer of protection in 
248 addition to the mechanical barrier of the foam itself. This additional protection could

249 potentially prevent chitin-degrading pathogens from damaging the eggs, as in the case of

250 the chironomid (Chironomus sp.) and Vibrio cholerae interaction (Broza \& Halpern

251 2001; Laviad et al., 2016). In the locust's case, it is possible that the gram-positive

252 targeted lysozyme (Raglan \& Criss 2017) in the foam is responsible for the absence of

253 Weissella (a firmicute) in the foam and offspring, despite its dominance in the maternal

254 gut samples. This is in contrast to Corynebacterium (an actinobacterium), which shows

255 resistance to lysozyme due to its unique mycolic acids containing an outer membrane

256 (Hirasawa et al. 2000, Toyoda et al. 2018), thus enabling it to proliferate in this

257 environment and to be transmitted to the hatchlings as they crawl through the foam.

258 We have demonstrated here that chitin is a major component of the locust foam plug. The 259 solid, hydrophobic nature of this polysaccharide probably may act as a physical 260 protective layer against predatory organisms as well as against desiccation. To the best

261 of our knowledge, such use of foam to protect an egg-mass, both mechanically and 262 immunologically, has been known to date only from a few foam-nesting frog species.

263 These frogs cover their eggs with a protein-rich foam to protect them from desiccation 264 and pathogens (Cooper \& Kennedy 2010).

265 In previous studies we had hypothesized that the desert locust employs some sort of 266 mechanism in order to inoculate its offspring with beneficial bacteria; and, accordingly, 267 that it maintains specific bacterial symbionts within the population across generations 268 (Lavy et al., 2019, 2020). Here we have provided evidence suggesting that the foam 269 deposited above the eggs has the potential to serve as such an inoculation mechanism. 270 Furthermore, we provide data indicating that the foam is more than merely a reservoir for 
271 the bacteria deposited by the mothers; it is also a selective medium, potentially

272 supporting mutualistic species while inhibiting the proliferation of others, thus

273 maintaining a beneficial bacterial consortium for transmission across locust generations.

274 We are far from fully understanding the contributions of microbes to locust biology; and

275 further in-vivo experiments, as well as exploration of the possible fungal and viral

276 symbionts residing within the locusts, are needed in order to elucidate these important

277 interactions. Nevertheless, the findings presented here provide important insights into a

278 little addressed aspect of the locust's ability to execute long-distance, trans-generational

279 devastating plagues.

\section{Experimental procedures:}

282 To test the hypothesis of symbiont vertical transmission, we chose to combine several 283 approaches. First, we employed 16S rRNA amplicon sequencing in order to determine

284 the bacterial composition of locust females, their offspring, the potential bacterial 285 inoculation vectors that constitute the eggs' immediate surroundings (in the present study 286 this was the sand in which the female oviposited), and the foam plug secreted onto the 287 egg pod itself (Fig. 1c). We then used an engineered bacterium to test for in-vivo 288 maternal inoculation. Finally, we applied a biochemical approach to analyze the foam's 289 composition, as a candidate vector for the symbionts' maternal inoculation.

290 Egg pod sampling 
291 Gregarious locusts were reared under crowded conditions for many consecutive 292 generations at $33 \pm 3^{\circ} \mathrm{C}$ and a photoperiod of $14 \mathrm{~L}: 10 \mathrm{D}$, and fed fresh wheat grass and dry 293 oats (for details see Lavy et al. 2019).

294 In order to examine the possibility of transgenerational bacterial transmission, we 295 conducted an experiment comparing the bacterial composition of locust mothers with that 296 of their newly-hatched and viable pre-hatched offspring.

297 Newly-matured females were introduced individually into11x12x14.5 cm metal cages 298 containing two mature males and food. A $50 \mathrm{ml}$ centrifuge tube (Corning, NY, United 299 States) filled with autoclaved moistened sand was replaced daily until oviposition 300 occurred and egg pods were detected. The females were then sacrificed and kept 301 individually in $70 \%$ absolute ethanol, at $-20^{\circ} \mathrm{C}$, until tissue sampling.

302 The tubes containing egg pods were incubated at $37^{\circ} \mathrm{C}$. Around day 11 of incubation (the 303 typical duration of embryonic development under these conditions) the tubes were 304 meticulously examined for the presence of hatchlings. Upon detection (within 30 min of 305 their emergence onto the sand surface) three hatchlings were collected into a 1.5 plastic 306 tube using sterile forceps (each pooled hatchling group is considered as one sample). The 307 egg pod was then excavated and three healthy-appearing locust-bearing eggs were 308 collected in the same manner.

309 The pooled hatchlings and the unhatched larvae were sacrificed, washed, and vortexed 310 five times $\mathrm{x} 1$ minute in filtered saline $(0.9 \% \mathrm{NaCl})$ to remove unattached external 311 bacteria (Fukatsu \& Hosokawa 2002), and stored in $70 \%$ absolute ethanol at $-20^{\circ} \mathrm{C}$ until 312 further use. Samples of the foam and the surrounding sand were also collected from each 
313 tube containing an egg pod and stored in the same manner, allowing us to assign hatched

314 larvae to their unhatched siblings and to the foam and the sand from the same tube (i.e.

315 the same egg pod).

316 Controlling for the foam as an inoculation source-with and without foam treatment

317 Sexually-mature females were housed individually and supplied with sand-filled

318 oviposition tubes as described above. Prior to autoclaving, the sand tubes were cut

319 longitudinally and then re-attached. After the female had laid eggs, the egg pods were

320 incubated at $37^{\circ} \mathrm{C}$ for five days. On day 5 post-oviposition, the tubes were opened along

321 the pre-made cut and some of the eggs were removed and placed in a fresh similar tube of

322 autoclaved sand. The original egg-containing tubes were resealed and incubated. The

323 eggs that had remained in the original tube gave rise to hatchlings that surfaced through

324 the foam plug (foam treatment), while the hatchlings emerging from the relocated eggs

325 surfaced only through a layer of moistened sand (without foam treatment). Upon

326 surfacing, the hatchlings were collected in groups of three, washed five times as

327 described above, and stored in $70 \%$ absolute ethanol at $-20^{\circ} \mathrm{C}$ until further use.

328 Gut sampling offemales

329 The locusts' wings and limbs were dissected out and their body surface was sterilized by

330 submerging in $1 \% \mathrm{NaOCl}$ solution for 2 min followed by two consecutive washings in

331 fresh double-distilled water. The bodies were then dissected aseptically (according to the

332 protocol detailed in Lavy et al. (2019)) and their hind-gut collected. The excised samples

333 were kept individually in $70 \%$ absolute ethanol at $-20^{\circ} \mathrm{C}$ until DNA extraction. 
335 Ethanol was removed and bacterial genomic DNA was extracted using the "Powersoil"

336 DNA isolation Kit (Mo Bio Laboratories Inc., Carlsbad CA, United States), according to

337 the manufacturer's instructions, using $60 \mu 1$ for final DNA elution. To determine bacterial

338 composition, polymerase chain reaction (PCR) of hypervariable areas V3 and V4 of the

339 prokaryotic 16S rRNA gene was performed on the extracted DNA; using a universal

340 primer containing 5-end common sequences (CS1-341F 5'-

341 ACACTGACGACATGGTTCTACANNNNCCTACGGGAGGC AGCAG and CS2-

342 806R 5'-TACGGTAGCAGAGACTTGG TCTGGACTACHVGGGTW TCTAAT). PCR

343 conditions: initial step of $94^{\circ} \mathrm{C}$ for $2 \mathrm{~min}$, followed by $30 \mathrm{PCR}$ cycles of denaturation at

$34494^{\circ} \mathrm{C}$ for $30 \mathrm{sec}$, annealing at $50^{\circ} \mathrm{C}$ for $30 \mathrm{sec}$ and extension at $72^{\circ} \mathrm{C}$ for $30 \mathrm{sec}$, ending the

345 reaction with 4 min at $72^{\circ} \mathrm{C}$. The reactions were performed using the PCR master mix Go

$346 \mathrm{Taq}^{\circledR}$ Green Master Mix (Promega Corporation, Madison, WI, United States). PCR

347 product validation was conducted by agarose gel $1 \%$ electrophoresis, and validated

348 samples were sent for deep sequencing of the amplified amplicons ( 430 bp per read),

349 conducted on an Illumina MiSeq platform at the Chicago Sequencing Center of the

350 University of Illinois. Tubes containing all reagents but lacking samples, were added to

351 every sequencing batch to rule out contamination.

352 Data analyses

353 Demultiplexed raw sequences were quality filtered (bases with a PHRED score $<20$ were 354 removed) and merged using PEAR (Zhang et al., 2014). Sequences of less than 380 bp 355 (after merging and trimming) were discarded. Data were then analyzed using the 356 Quantitative Insights Into Microbial Ecology (QIIME) package (Caporaso et al., 2010) 357 and Vsearch (Rognes et al., 2016) was used for chimera detection and elimination. 
358 Merged and trimmed data were additionally analyzed with the DADA2 pipeline

359 (Callahan et al., 2016) to infer exact sequences for amplicon sequence variant (ASV)

360 analyses. Chloroplast and E-coli sequences were excluded from the downstream analysis

361 due to the known gut content, and to our inability to completely avoid Master Mix

362 derived E-coli fragments. Average read depth was 21,910 seqs/sample (range 1-56,830).

363 Data were rarified to 980 seqs/sample prior to analysis. The "with foam" and "without 364 foam" sections were analyzed separately, and the data of this part were rarefied to 1100

365 seqs/sample. All statistical analyses were conducted using R v.3.4.1. (R core team 2013).

366 Bray-Curtis based Analysis of similarities-Anosim, principal coordinate analysis (PCoA),

367 and Spearman's rank correlations were carried out using the vegan 2.4-3 package

368 (Oksanen et al., 2008).

In-vivo foam inoculation

370 We used a strain of Klebsiella pneumoniae isolated from locust females and introduced it

371 with two different antibiotic resistance markers to test for in-vivo bacterial transmission

372 from the female gut to the egg pod and its surroundings, according to the following steps:

374 Locust-isolated K. pneumoniae (top hit type-strain: ATCC 13884(T), similarity: 99.76\%),

375 grown overnight, was inoculated into a fresh lysogeny broth (LB) and incubated at $37^{\circ} \mathrm{C}$

376 for $90 \mathrm{~min}$. The cells were then chilled on ice, harvested by two rounds of centrifugation

377 (4000 rpm for $10 \mathrm{~min}$ ) and washed with double-distilled water, followed by resuspension

378 in $10 \%$ glycerol. $60 \mu \mathrm{l}$ of cell suspension was mixed with $2 \mu \mathrm{l}$ of pUA66 (a non-

379 conjugative, low-copy plasmid, Zaslaver et al., 2004) and electroporated. The cells were 
380 then transferred into $1 \mathrm{ml} \mathrm{LB}$ broth and incubated at $37^{\circ} \mathrm{C}$ for $1 \mathrm{~h}$, followed by plating on

381 a selective substrate (final concentration of kanamycin: $50 \mu \mathrm{g} / \mathrm{L}$ ) to select for the

382 resistant phenotype of pUA66.

2. Streptomycin resistant mutation induction:

$1 \mathrm{ml}$ of pUA66 harboring $K$. pneumoniae LB culture was centrifuged, washed, and re-

386 suspended in saline $0.9 \% \mathrm{NaCl} .100 \mu \mathrm{l}$ was then applied onto LB plates containing both

387 kanamycin $(50 \mu \mathrm{g} / \mathrm{ml})$ and streptomycin. $(100 \mu \mathrm{g} / \mathrm{ml})$. A K. pneumoniae colony that had

388 been formed during overnight incubation at $37^{\circ} \mathrm{C}$ was applied onto a fresh selective plate 389 to maintain the resistant strain.

391 The engineered bacteria were cultured in antibiotic-free LB at $37^{\circ} \mathrm{C}$. Every 24 hours 100 $392 \mu \mathrm{l}$ were transferred to a fresh $2 \mathrm{ml} \mathrm{LB}$ medium and an additional $100 \mu \mathrm{l}$ were applied 393 onto selective plates overnight. Suspected Klebsiella colonies from each plate were 394 boiled for $10 \mathrm{~min}$ in $20 \mu \mathrm{l}$ of double-distilled water. $1 \mu \mathrm{l}$ of the boiled mixture was then 395 used for diagnostic PCR with specific pUA66 primers (forward primer, 5'396 CATAAGATGAGCCCAAG-3; reverse primer, 5'-GTCAGTACATTCCCAAGG-3) to 397 verify pUA66 presence. PCR conditions were: initial step of $94^{\circ} \mathrm{C}$ for 2 min, followed by 398 thirty PCR cycles of denaturation at $94^{\circ} \mathrm{C}$ for $30 \mathrm{~s}$, annealing at $50^{\circ} \mathrm{C}$ for 30 s and 399 extension at $72^{\circ} \mathrm{C}$ for 30 s. Ending the reaction with 4 min at $72^{\circ} \mathrm{C}$. The enzyme used in

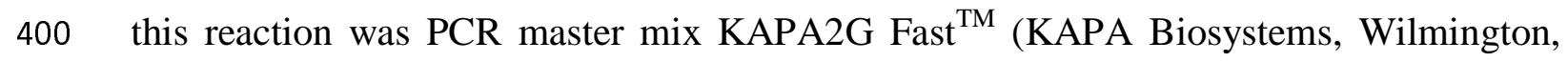
401 MA, United States). 
403 Fifteen mature female locusts were force-fed with $50 \mu$ l saline containing $\sim 615$ X $10^{6} \mathrm{~K}$.

404 pneumoniae cells and transferred together to a female-only cage. One-week post-

405 inoculation fecal pellets were collected from each individual female, applied onto a

406 selective MacConkey agar, incubated for $24 \mathrm{~h}$ at $37^{\circ} \mathrm{C}$, and diagnostic PCR for pUA66

407 presence was conducted on Klebsiella-suspected colonies from each plate to confirm the

408 presence of the engineered bacteria in the females' gut.

409 The females were then transferred individually to $11 \times 12 \times 14.5 \mathrm{~cm}$ metal cages containing

410 two mature males, and fresh tubes of autoclaved sand and food were replaced daily.

411 Within 24 hours of oviposition, a sample of the foam plug $(\mathrm{n}=15)$ and the surrounding

412 tube-sand $(\mathrm{n}=15)$ was diluted and streaked on selective agar MacConkey plates, and

413 incubated at $37^{\circ} \mathrm{C}$ for 24 h. pUA66 diagnostic PCR was performed on Klebsiella-

414 suspected colonies from each plate.

$415 \quad$ Foam biochemical analysis

416 Mature female locusts were kept individually in metal cages containing two mature

417 males. As oviposition substrate they were provided with a $50 \mathrm{ml}$ centrifuge tube

418 containing chemically inert glass beads (diameter: $1 \mathrm{~mm}$ ) (Paul Marienfeld GmbH \& Co.

419 KG, Lauda-Königshofe, Germany) saturated with double-distilled water.

420 The tubes were replaced daily and, when oviposition occurred, the egg pod was collected

421 and its foam plug was removed and washed in double-distilled water to remove the glass

422 beads. The foam was then left for 24 hours to dry at $4^{\circ} \mathrm{C}$ and the dry foam was kept at

423 room temperature until chemical analysis. 
424 For elemental analysis measurements, the foam plugs were crushed manually, the glass

425 beads were removed, and the crushed matrix was soaked in $0.5 \mathrm{M} \mathrm{NaOH}$ in $25^{\circ} \mathrm{C}$ until

426 complete decomposition (clear yellowish solution). The soluble hydrolysis products were

427 filtered from the beads, lyophilized, and analyzed. The nitrogen content was converted to 428 protein according to the Nitrogen-to-Protein conversion factor range for insects reported 429 by Janssen et al. (2017).

430 In order to analyze the foam protein content, $1.5 \mathrm{~g}$ of crushed foam plugs (with the glass 431 beads) were mixed with $8 \mathrm{ml}$ ethanol and sealed in a $14 \mathrm{ml}$ glass vial. The mixture was 432 incubated at $60^{\circ} \mathrm{C}$ for $96 \mathrm{~h}$ until the foam had almost completely decomposed. Ethanol 433 evaporation was performed in an $80^{\circ} \mathrm{C}$ dry bath and the pellet was dissolved in reducing 434 sample buffer (containing $\beta$-mercaptoethnol) for SDS-PAGE analysis. The sample was 435 boiled at $100^{\circ} \mathrm{C}$ for $10 \mathrm{~min}$. Finally, SDS-PAGE analysis was performed with $15 \%$ 436 acrylamide gel at $90 \mathrm{~V}$ for $4 \mathrm{~h}$. The six main identified bands (Fig. S1 in supporting 437 information) were analyzed by Mass-Spectrometry (MS) in the Smoler Protein Research 438 Center at the Technion, Haifa. The screening of protein results was performed against the 439 Acrididae and Locusta protein data-bases. Only identified peptides that passed the False 440 Discovery Rate (FDR) correction with a 99\% confidence interval were used for further 441 analysis.

442 Since the samples could not be dissolved in a manner that would enable carbohydrate 443 analysis, we incubated $0.04 \mathrm{~g}$ of sand-coated foam plugs in $50 \mathrm{mM}$ potassium phosphate 444 (pH 6.0) buffer with decreasing chitinase concentrations (Sigma- Aldrich, St. Louis, MO, 445 USA), in order to determine chitin or chitin-like presence in the foam.

\section{Acknowledgments}


447 We would like to express the authors' gratitude to Dr. Leah Reshef for her informed 448 suggestions and her important help in data analyses.

449 References

450 Broza, M. \& Halpern, M. (2001). Pathogen reservoirs: Chironomid egg masses and vibrio $451 \quad$ cholerae. Nature, 412, 40.

452 Callahan, B.J., McMurdie, P.J., Rosen, M.J., Han, A.W., Johnson, A.J.A. \& Holmes, S.P. 453 (2016). DADA2: High resolution sample inference from Illumina amplicon data. $454 \quad$ Nat. Methods, 13, 581-583.

455 Caporaso, J.G., Kuczynski, J., Stombaugh, J., Bittinger, K., Bushman, F.D., Costello, 456 E.K., et al. (2010). QIIME allows analysis of high-throughput community 457 sequencing data. Nat. Methods, 7, 335-6.

458 Cease, A.J., Elser, J.J., Fenichel, E.P., Hadrich, J.C., Harrison, J.F. \& Robinson, B.E. 459 (2015). Living with locusts: Connecting soil nitrogen, locust outbreaks, livelihoods, and livestock markets. Bioscience, 65, 551-558.

461 Charnley, A.K., Hunt, j. \& Dillon, R.J. (1985). The germ-free culture of desert locusts, Schistocerca gregaria. J. Insect Physiol, 6, 477-485.

463 Cooper, A. \& Kennedy, M.W. (2010). Biofoams and natural protein surfactants. Biophys. $464 \quad$ Chem., 151, 96-104.

465 Dillon, R.J. \& Charnley, A.K. (2002). Mutualism between the desert locust Schistocerca 466 gregaria and its gut microbiota. Res. Microbiol., 153, 503-509.

467 Dillon, R.J. \& Charnley, A.K. (1995). Chemical barriers to gut infection in the desert 
locust - in-vivo production of antimicrobial phenols associated with the bacterium Pantoea agglomerans. J. Invertebr. Pathol., 66, 72-75.

470 Dillon, R.J., Vennard, C.T., Buckling, A. \& Charnley, A.K. (2005). Diversity of locust 471 gut bacteria protects against pathogen invasion. Ecol. Lett., 8, 1291-1298.

472 Dillon, R.J., Vennard, C.T. \& Charnley, A.K. (2000). Exploitation of gut bacteria in the 473 locust. Nature, 403, 851-852.

474 Engl, T., Kroiss, J., Kai, M., Nechitaylo, T.Y., Svatoš, A. \& Kaltenpoth, M. (2018). 475 Evolutionary stability of antibiotic protection in a defensive symbiosis. Proc. Natl. $476 \quad$ Acad. Sci., 201719797.

477 Fukatsu, T. \& Hosokawa, T. (2002). Capsule-transmitted gut symbiotic bacterium of the Japanese common plataspid stinkbug, megacopta punctatissima. Appl. Environ. Microbiol., 68, 389-396.

Hägele, B.F., Oag, V., Bouaïchi, A., McCaffery, A.R. \& Simpson, S.J. (2000). The role of female accessory glands in maternal inheritance of phase in the desert locust Schistocerca gregaria. J. Insect Physiol., 46, 275-280. 
growth, and L-glutamate production. J. Bacteriol, 182, 2696-2701.

490

491

492

493

494

495

496

497

498

499

500

501

502

503

504

505

Van Huis, A., Cressman, K. \& Magor, J.I. (2007). Preventing desert locust plagues: Optimizing management interventions: Mini review. Entomol. Exp. Appl.

Itoh, N., Morihama, R., Wang, J., Okada, K. \& Mizuguchi, N. (1997). Purification and characterization of phenylacetaldehyde reductase from a styrene-assimilating Corynebacterium strain, ST-10. Appl. Environ. Microbiol., 63, 3783-3788.

Itoh, N., Yoshida, K. \& Okada, K. (1996). Isolation and Identification of Styrenedegrading Corynebacterium Strains, and Their Styrene Metabolism. Biosci. Biotechnol., 60, 1826-1830.

Janssen, R.H., Vincken, J.-P., Broek, L.A.M. van den, Fogliano, V. \& Lakemond, C.M.M. (2017). Nitrogen-to-Protein Conversion Factors for Three Edible Insects: Tenebrio molitor, Alphitobius diaperinus, and Hermetia illucens.

Kaltenpoth, M., Schmitt, T., Polidori, C., Koedam, D. \& Strohm, E. (2010). Symbiotic streptomycetes in antennal glands of the South American digger wasp genus Trachypus (Hymenoptera, Crabronidae). Physiol. Entomol., 35, 196-200.

Kaltenpoth, M., Wolfgang, G., Gudrun, H. \& Erhard, S. (2005). Symbiotic Bacteria Protect Wasp Larvaefrom Fungal Infestation. Curr. Biol., 15, 475-479.

Kroiss, J., Kaltenpoth, M., Schneider, B., Schwinger, M.G., Hertweck, C., Maddula, R.K., et al. (2010). Symbiotic streptomycetes provide antibiotic combination prophylaxis for wasp offspring. Nat. Chem. Biol., 6, 261-263.

Laviad, S., Golan, A., Shaked, T., Vaizel-Ohayon, D., Halpern, M. \& Pick, E. (2016). 
511 Lavy, O., Gophna, U., Gefen, E. \& Ayali, A. (2019). The effect of density-dependent 512 phase on the locust gut bacterial composition. Front. Microbiol., 10, 1-8.

513 Lavy, O., Gophna, U., Gefen, E. \& Ayali, A. (2020). Dynamics of bacterial composition 514 in the locust reproductive tract are affected by the density-dependent phase. FEMS $515 \quad$ Microbiol. Ecol, 96, fiaa044.

516 [dataset] Lavy, O., Gophna, U., Gefen, E. \& Ayali, A. (2020). Locust bacterial

517 symbionts. SRA archive. https://dataview.ncbi.nlm.nih.gov/object/PRJNA598984

518 Lorenz, M.W. (2009). Migration and trans-Atlantic flight of locusts. Quat. Int., 196, 4$519 \quad 12$.

520 Oksanen, J., Kindt, R., Legendre, P., O’Hara, B., Simpson, G.L., Solymos, P.M., et al. 521 (2008). The vegan package. Community Ecol. Packag., 190.

522 Onchuru, T.O., Martinez, A., Ingham, C.S. \& Kaltenpoth, M. (2018). Transmission of 523 mutualistic bacteria in social and gregarious insects. Curr. Opin. Insect Sci., 28, 50$524 \quad 58$.

525 Park, R., Dzialo, M.C., Spaepen, S., Nsabimana, D., Gielens, K., Devriese, H., et al. 526 (2019). Microbial communities of the house fly Musca domestica vary with 527 geographical location and habitat. Microbiome, 7, 1-12.

528 Pener, M.P. \& Simpson, S.J. (2009). Locust phase polyphenism: an update. Adv. In Insect $529 \quad$ Phys., 36, 1-272.

530 Popov, G.B. (1958). Ecological Studies on Oviposition by Swarms of the Desert Locust 
(Schistocerca gregaria Forskal) in eastern Africa. Anti-Locust Bull., 70.

532 Ragland, S.A. \& Criss, A.K. (2017). From bacterial killing to immune modulation:

533 Recent insights into the functions of lysozyme. PLoS Pathog.

534 Rai, M.M., Hassanali, A., Saini, R.K., Odongo, H. \& Kahoro, H. (1997). Identification of 535 components of the oviposition aggregation pheromone of the gregarious desert locust, Schistocerca gregaria (Forskal). J. Insect Physiol., 43, 83-87.

537 Rognes, T., Flouri, T., Nichols, B., Quince, C. \& Mahé, F. (2016). VSEARCH: a 538 versatile open source tool for metagenomics. PeerJ Prepr., 4, e2409v1.

539 Salem, H., Florez, L., Gerardo, N. \& Kaltenpoth, M. (2015). An out-of-body experience: 540 the extracellular dimension for the transmission of mutualistic bacteria in insects. Proc. R. Soc. B Biol. Sci., 282, 20142957-20142957.

542 Segata, N., Baldini, F., Pompon, J., Garrett, W.S., Truong, D.T., Dabiré, R.K., et al. 543 (2016). The reproductive tracts of two malaria vectors are populated by a core 544 microbiome and by gender- and swarm-enriched microbial biomarkers. Sci. Rep., 6, $545 \quad 24207$.

546 Senderovich, Y., Gershtein, Y., Halewa, E. \& Halpern, M. (2008). Vibrio cholerae and 547 Aeromonas: do they share a mutual host? ISME J., 2, 276-283.

548 Shi, W., Guo, Y., Xu, C., Tan, S., Miao, J., Feng, Y., et al. (2014). Unveiling the 549 mechanism by which microsporidian parasites prevent locust swarm behavior. Proc. $550 \quad$ Natl. Acad. Sci. U. S. A., 111, 1343-8.

551 Shukla, S.P., Vogel, H., Heckel, D.G., Vilcinskas, A. \& Kaltenpoth, M. (2017). Burying 

to their offspring.

Skaf, R., Popov, G.B. \& Roffey, J. (1990). The desert locust: an international challenge. Philos. Trans. - R. Soc. London, B, 328, 525-538.

Soussi, A., Ferjani, R., Marasco, R., Guesmi, A., Cherif, H., Rolli, E., et al. (2015). Plantassociated microbiomes in arid lands: diversity, ecology and biotechnological potential. Plant Soil, 405, 357-370.

Symmons, P.M. \& Cressman, K. (2001). Desert Locust Guidelines., 42.

Tobias, N.J. (2016). Insect Vectors of Disease: Untapped Reservoirs for New Antimicrobials? Front. Microbiol., 7, 1-5.

Toyoda, K. \& Inui, M. (2018). Extracytoplasmic function sigma factor $\sigma D$ confers resistance to environmental stress by enhancing mycolate synthesis and modifying

Wang, Y. \& Rozen, D.E. (2017). Gut Microbiota Colonization and Transmission in the peptidoglycan structures in Corynebacterium glutamicum. Mol. Microbiol, 107, Burying Beetle Nicrophorus vespilloides throughout Development.

Wang, Y. \& Rozen, D.E. (2018). Gut microbiota in the burying beetle, Nicrophorus vespilloides, provide colonization resistance against larval bacterial pathogens. Ecol. (2004). Just-in-time transcription program in metabolic pathways. 
573 Zhang, L., Lecoq, M., Latchininsky, A. \& Hunter, D. (2019). Locust and Grasshopper

574 Management. Annu. Rev. Entomol, 64, 15-34.

575 Zhang, J., Kobert, K., Flouri, T. \& Stamatakis, A. (2014). PEAR: A fast and accurate 576 Illumina Paired-End reAd mergeR. Bioinformatics, 30, 614-620.

577 Zucchi, T.D., Prado, S.S. \& Cônsoli, F.L. (2012). The gastric caeca of pentatomids as a 578 house for actinomycetes. BMC Microbiol., 12, 1-7.

\section{Author contributions}

581 OL, AA, and EG conceived the study and designed the experiments. OL conducted the 582 experiments and analyzed the data. UG oversaw the microbial ecology experiments and 583 guided data analysis. AF and SG conducted the biochemical analyses. OL wrote the first 584 draft of the manuscript, and all authors contributed substantially to the revisions.

\section{Legends:}

586 Table 1: Bacterial amplicon sequence variants (ASVs) shared among samples of locust 587 females, the foam plug secreted by those specific females, and the hatchlings emerging 588 through this foam (only full sets of samples are presented, $n=14$ sets). The bacterium 589 Corynebacterium (bold) is shared among 10 of the 14 data sets.

590 Table 2: Elemental analysis of locust nest plugs.

592 Table 3: Proteins found in the foam plug and suspected to constitute immunity593 conferring agents that may play a bacterial regulatory role in the foam plug. This is a 
594 partial list of 8 out of 40 proteins. Only immunity-related proteins are presented here. The

595 full protein list can be found in Table S3.

597 Figure 1: Illustrations showing (a) A female locust extending its abdomen while digging

598 in order to lay eggs in the soil and laying eggs up to $14 \mathrm{~cm}$ deep in the soil. (b) The egg

599 pod is enveloped in a secretion of foam. (b1) The foam sheath surrounding the eggs,

600 partially removed to illustrate the foam plug above the eggs.

601

602 Figure 2: Genus-level, Shannon biodiversity indexes of the bacterial composition of the

603 hatched and unhatched siblings, as well as the foam and sand surrounding the eggs.

604 Differences between the groups were analyzed by Kruskal-Wallis test and Dunn's post-

605 hoc test. Boxes within the violin represent the range containing $50 \%$ of the data points, 606 and the red horizontal lines denote the median.

607 Figure 3: (a) Relative abundance of the core bacterial genera (present in minimum 80\% 608 of the samples of a specific tissue) in a female's hindgut (left, $n=16$ ), in the hatchlings 609 emerging through the foam (right, $n=16$ ), and in the foam (bottom, $n=15$ ). Inserts present 610 a sketch of the sample origin (highlighted in white). Bars representing bacteria noted in 611 the main text are colored. (b) Venn diagram of the dominant shared ASVs (> 75 reads per 612 sample type) among the female's hindgut, the hatchlings, and the foam (background color 613 as in a, above). Taxonomical assignment of the 23 shared sequences is listed to the right 614 of the diagram. 
615 Figure 4: Graphical representation of the significant correlation of Corynebacterium 616 relative abundance in the foam and in the hatchlings surfacing through that foam 617 (Spearman rho: $p=0.005, \mathrm{r}=0.67, \mathrm{n}=15$ ). Dots represent foam and hatchlings from the 618 same mother, and the gray area stands for the standard error.

619 Figure 5: Relative abundance comparison of Corynebacterium in locust hatchlings 620 surfacing in the with-foam and in the without-foam treatments. (Mann Whitney. $621 P<0.001)$.

622 Figure 6: Partial hydrolysis of the foam plug mass by incubation with decreasing order of 623 chitinase active unit (U) concentrations.

624 Figure S1: SDS-PAGE visualization of proteins extracted from the locust foam plug.

625 Two lanes represent duplicates and red squares represent the band numbering used for

626 MS analysis.

627 Figure S2: Relative abundance of the core bacterial genera (minimum of $80 \%$ presence 628 in the tissue samples) of the pre-hatching samples $(n=11)$; and the sand samples $(n=16)$.

629 Bacteria noted in the main text are uniformly colored.

630 Table S3: Full protein profile of the foam plug. 


\section{Table 1:}

633

\begin{tabular}{ccc} 
Female ID & $\begin{array}{c}\text { Number of shared } \\
\text { ASVs }\end{array}$ & $\begin{array}{c}\text { Assigned genera } \\
\text { non }\end{array}$ \\
\hline 1 & 2 & $\begin{array}{c}\text { *Corynebacterium } \\
\text { Pseudocitrobacter } \\
\text { Corynebacterium }\end{array}$ \\
3 & 3 & Paracoccus \\
& & $\begin{array}{c}\text { Brevibacterium } \\
\text { Corynebacterium } \\
\text { Corynebacterium }\end{array}$ \\
4 & 1 & $\begin{array}{c}\text { Corynebacterium } \\
\text { Stenotrophomonas } \\
6\end{array}$ \\
6 & 1 & $\begin{array}{c}\text { Corynebacterium } \\
\text { Brachybacterium }\end{array}$ \\
7 & 2 & Klebsiella \\
8 & 1 & na
\end{tabular}

10

4

Corynebacterium

Klebsiella (x2)

Staphylococcus

11

3

Corynebacterium

Micrococcus

Pelomonas

12

Corynebacterium

$$
\text { Klebsiella }
$$

13

non

na

14

4

\section{Corynebacterium}

Enterobacter

Brevibacterium

Enterococcus

* The Corynebacterium assigned ASV, is identical throughout the presented data. 


\section{Table 2:}

\begin{tabular}{ccccc} 
Repeat & $\mathbf{N}(\%)$ & $\mathbf{C}(\%)$ & $\mathbf{H}(\%)$ & $\mathbf{S}(\boldsymbol{6} \%)$ \\
\hline A & 0.741 & 2.840 & 0.358 & 0.0845 \\
B & 0.519 & 3.540 & 0.129 & 0637 \\
\hline
\end{tabular}

638

\section{Table 3:}

*Accession Description MW [kDa] Band in Fig. S1 Reference

\begin{tabular}{ccccc}
\hline 40949967 & $\begin{array}{c}\text { Thaumatin-like } \\
\text { protein }\end{array}$ & 26.00 & 3 & Brandazza et al. 2004 \\
1120618827 & Vitellogenin A & 150.71 & 6 & Song et al. 2013 \\
1394299014 & C-type lysozyme & 79.99 & 6 & Unpublished \\
972988174 & Ferritin subunit & 26.53 & 4 & Bonilla et al. 2015 \\
414079973 & Prophenoloxidase 1 & 84.20 & 1 & Unpublished \\
1231943145 & Vitellogenin B & 26.45 & 6 & Unpublished \\
414145764 & Chain C, Greglin & 9.18 & 3 & Derache et al. 2012 \\
371942914 & C-type lysozyme & 15.69 & 1 & Mohamed et al. 2016 \\
\hline
\end{tabular}

* NCBI protein-accession number

640 


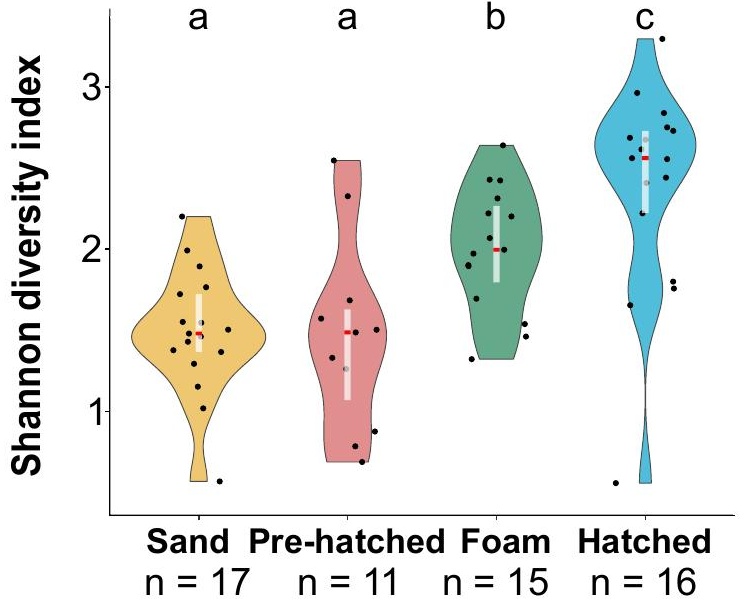



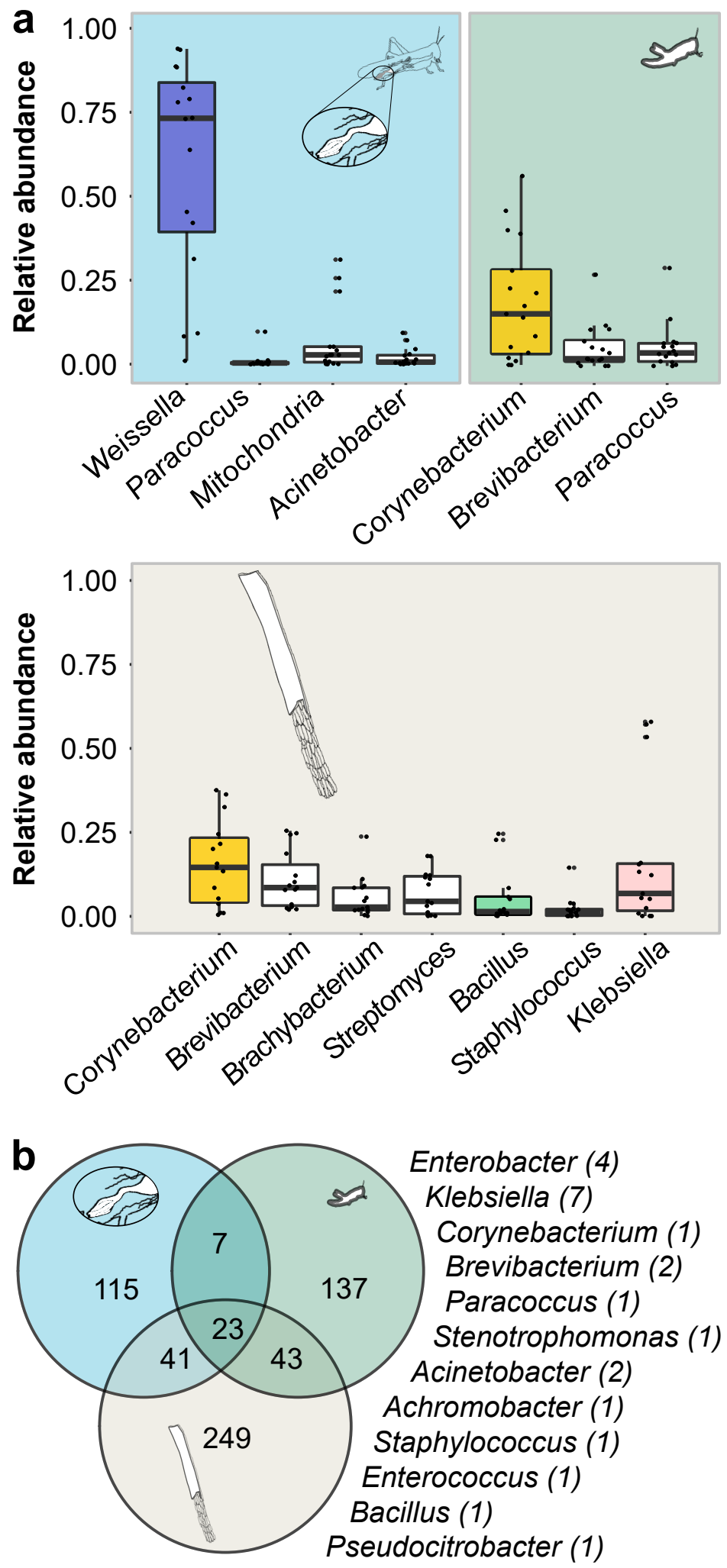


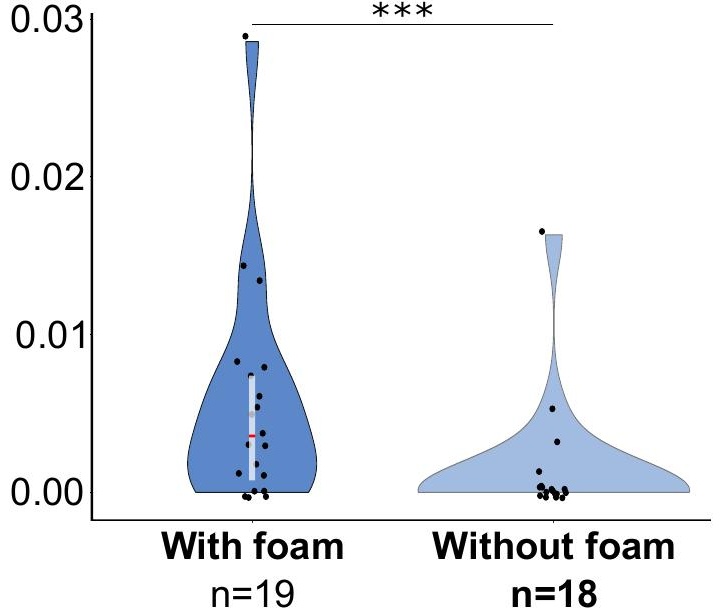




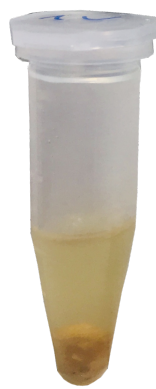

